\title{
Les Complications Osseuses au Cours de la Drepanocytose au Service d'Hematologie-Oncologie du CHU de Donka de Conakry
}

\author{
Diakite Mamady, \\ Kante Ansoumane Sayon,
}

Assistant Chef Clinique d'Hématologie, Service d'Hémato-Oncology

de l'Hôpital National Donka/CHU de Conakry, Guinée

Dambakate Alhassane,

Médecin Généraliste au Service d'Hémato-Oncology de l'Hôpital National

Donka/CHU de Conakry, Guinée

Bathily Moussa,

Assistant Chef Clinique d'Hématologie, Hôpital Point G de Bamako, Mali

Camara Djongbe,

Médecin Généraliste au Service d'Hémato-Oncology de l'Hôpital National

Donka/CHU de Conakry, Guinée

Tolo-Diebkile Aissata,

Professeur Titulaire d'Hematologie Clinique, CHU de Yopougon d'Abidjan,

Côte d'Ivoire

Doi: 10.19044/esj.2019.v15n12p252 URL:http://dx.doi.org/10.19044/esj.2019.v15n12p252

Résumé

Introduction : la drépanocytose a une évolution caractérisée par des complications aiguës et chroniques qui intéressent presque tous les organes cibles de l'organisme (os, yeux, cœur, reins, cerveau ...). L'objectif de cette étude était de décrire les complications osseuses de la drépanocytose. Patients et Méthodes : Il s'agissait d'une étude rétrospective réalisée au service d'Hématologie - Oncologie du CHU de Donka qui s'est étalée sur une période de cinq ans (5ans) allant du $1^{\mathrm{er}}$ janvier 2011 au 31 décembre 2015 Résultats : La fréquence des complications osseuses de la drépanocytose était de $15 \%$. Il y avait une prédominance du sexe féminin avec le sex-ratio de 0,8 . L'âge moyen des patients était de 27,5 ans avec des extrêmes de 10 et de $\quad 50$ ans. Le principal motif de consultation était la douleur osseuse retrouvée chez $100 \%$ de nos patients. L'ostéomyélite était la complication la plus fréquente soit $55,56 \%$ suivie de la nécrose de la tête fémorale soit $44,44 \%$. Conclusion : Il est ressorti de l'étude que la prévalence des complications osseuses est non négligeable. Une étude prospective et 
analytique avec un échantillon plus élevé serait nécessaire pour identifier les facteurs de risque et élaborer un protocole de prise en charge.

Mots-clés : Complications osseuses ; Drépanocytose ; CHU Donka, Conakry (Guinée)

\title{
Bone Complications During Drepanocytosis at the Hematology-Oncology Service of Donka / Conakry Hospital
}

\author{
Diakite Mamady, \\ Kante Ansoumane Sayon,
} Assistant Chef Clinique d'Hématologie, Service d'Hémato-Oncology de l'Hôpital National Donka/CHU de Conakry, Guinée

Dambakate Alhassane, Médecin Généraliste au Service d'Hémato-Oncology de l'Hôpital National Donka/CHU de Conakry, Guinée

Bathily Moussa,

Assistant Chef Clinique d'Hématologie, Hôpital Point G de Bamako, Mali

\section{Camara Djongbe,}

Médecin Généraliste au Service d'Hémato-Oncology de l'Hôpital National Donka/CHU de Conakry, Guinée

Tolo-Diebkile Aissata,

Professeur Titulaire d'Hematologie Clinique, CHU de Yopougon d'Abidjan, Côte d'Ivoire

\begin{abstract}
Introduction: Sickle cell disease has an evolution characterized by acute and chronic complications that affect almost all the target organs of the body (bones, eyes, heart, kidneys, brain ...). The purpose of this study was to describe the bone complications of sickle cell disease. Patients and Methods: This was a retrospective study performed in the HematologyOncology Department of the Donka Teaching Hospital over a five-year period (5 years) from 1 January 2011 to 31 December 2015. Results: The frequency of bone complication of sickle cell disease was $15 \%$. There was a predominance of the female sex with the sex ratio of 0.8 . The average age of the patients was 27.5 years with extremes of 10 and 50 years. The main reason
\end{abstract}


for consultation was bone pain found in $100 \%$ of our patients. Osteomyelitis was the most frequent $55.56 \%$, followed by Necrosis of the femoral head 44.44\%. Conclusion: The study found that the prevalence of bone complications is not negligible. A prospective and analytical study with a larger sample would be required to identify risk factors and develop a management protocol.

Keywords: Bone complications; Sickle cell disease; Donka Hospital, Conakry (Guinea)

\section{Introduction}

La drépanocytose est une hémoglobinopathie liée à une anomalie de structure la chaîne bêta de la globine. Cette anomalie se traduit par un remplacement de l'acide glutamique par la valine en position six sur la chaîne bêta de la globine. La drépanocytose est une maladie très répandue dans le monde (Cécile, 2012). Près de 120 millions de personnes dans le monde seraient porteuses d'une mutation drépanocytaire (Coulibaly et coll, 2010). La drépanocytose ne devient symptomatique qu'à l'état homozygote (SSFA2) ou hétérozygote composite (SC, S bêta thalassémie).

Véritable maladie invalidante, la drépanocytose a une évolution caractérisée par des complications aiguës et chroniques qui intéressent presque tous les organes nobles de l'organisme (os, articulations, cœur, reins, ...).

Les complications ostéo-articulaires seraient les plus fréquentes dans le monde (Anoosha, 2004). Elles peuvent être infectieuses (ostéomyélite essentiellement) ou ischémiques (ostéo- nécrose aseptique épiphysaire).

Elles atteignent presque tous les os et surviennent généralement de façon bilatérale et symétrique (Catonné et coll, 2004). La nécrose de la tête fémorale semble être la plus grave sur le plan fonctionnel. La goutte, l'arthrite septique, les arthropathies aiguës, l'hémarthrose et les polyarthrites chroniques destructives sont quelques complications observées dont la prise en charge est difficile. (Cécile, 2012).

Le polymorphisme clinique ;

Le nombre de cas devenant de plus en plus important

Les difficultés de diagnostic ont été les principaux motifs de cette étude dont le but était de rechercher les complications osseuses de la drépanocytose.

\section{Patients et Methodes}

Il s'agissait d'une étude rétrospective de type descriptif, réalisée au service d'Hématologie - Oncologie du CHU de Donka qui a portée sur une période de cinq ans (5ans) allant du $1^{\text {er }}$ janvier 2011 au 31 décembre 2015. L'étude a concerné tous les dossiers de malade des drépanocytaires au niveau 
desquels les complications osseuses ont été diagnostiquées selon les critères cliniques, biologiques et radiologiques.

Les paramètres de l'étude étaient: fréquence, tranche d'âge, sexe, motifs de consultation, type de complication, type de la drépanocytose et anomalie quantitative de l'hémogramme.

\section{Resultats}

Sur un échantillon de 120 patients colligés dans la période d'étude, 18 étaient porteurs d'une complication osseuse soit 15\%. L'âge moyen était de 27,5 \pm 9 avec les extrêmes de 10 et 50 ans. Nous avons noté une légère prédominance féminine $(55,56 \%)$ avec un Sex Ratio H/F de 0,8.

Les motifs de consultation étaient dominés par les douleurs osseuses quasi constantes chez tous nos patients (Tableau I). Deux complications ont marquées notre série : l'ostéomyélite $(55,56 \%)$ et la nécrose aseptique de la tête fémorale $(44,44 \%)$ dont 5 sur 8 cas de nécroses étaient bilatérales. Le fémur était l'os le plus atteint $(66,67 \%)$, tibia $(27,78 \%)$ et l'humérus $(5,55 \%)$.

Concernant la forme de drépanocytose, la drépanocytose majeure homozygote SSFA2 était de loin la plus fréquente avec 77,77\% (tableau II). L'hémogramme réalisé chez nos patients a retrouvé une polynucléose neutrophile $(55,56 \%)$, un cas de neutropénie et l'anémie toujours présente.

Tableau I : Répartition de nos patients selon les motifs de consultation

\begin{tabular}{llc}
\hline Motifs de consultation & Nombre de cas & Pourcentage $\%$ \\
\hline Douleurs osseuses & 18 & 100 \\
Fistulisation & 1 & 5,55 \\
Plaie chronique & 5 & 27,77 \\
Impotence fonctionnelle & 4 & 22,22 \\
Fièvre & 11 & 61,11 \\
\hline
\end{tabular}

Tableau II: Repartition de nos patients selon les types de drépanocytose

\begin{tabular}{lll}
\hline Types & Nombre de cas & Pourcentage \\
\hline SSFA2 & 14 & 77,77 \\
SFA2 & 1 & 5,55 \\
SC & 2 & 11,11 \\
SAFA2 & 1 & 5,55 \\
Total & $\mathbf{1 8}$ & $\mathbf{1 0 0}$ \\
\hline
\end{tabular}

\section{Discussion}

$\mathrm{Au}$ cours de cette étude, sur 120 dossiers drépanocytaires, 18 cas de complications osseuses ont été retrouvés soit une fréquence de $15 \%$. Ce résultat était inférieur à celui apporté par CATONNÉ Y et coll [4] où dans leur étude $28 \%$ des patients présentaient des lésions osseuses. Cette différence pourrait s'expliquer par le fait que notre étude a concerné uniquement les 
dossiers des sujets adultes d'une part. Certains drépanocytaires avec des lésions osseuses consultent dans d'autres services d'autre part. Les complications ostéoarticulaires de la drépanocytose sont multiples et peuvent se manifester sur le mode aigu (infarctus osseux, ostéomyélite aigue) ou chronique (ostéonécrose, ostéomyélite chronique). Elles ont un mécanisme commun, lié aux particularités physico-chimiques de l'hémoglobine $S$ (Habibi, 2004).

Nous avons noté une prédominance du sexe féminin avec un sex-ratio de 0,8 . Ce résultat est contraire à celui de N'DRI K et coll (N'dri, 2000) où il $\mathrm{y}$ avait une légère prédominance du sexe masculin. Cependant le sexe ne constitue pas un facteur de risque de survenu de complications osseuses.

L'âge moyen des patients était de 27,5 ans \pm 9 avec des extrêmes de 10 et de 50 ans. Notre résultat était différent de celui de Anoosha $\mathrm{H}$ et coll (Anoosha et coll, 2004) où l'âge moyen était de 16,5ans. Cette différence s'expliquerait par le fait que les cas des nourrissons et petits enfants ont été pris en charge ailleurs.

Le principal motif de consultation était la douleur osseuse retrouvée chez $100 \%$ de nos patients. L'ostéomyélite était la complication la plus fréquente soit 55,56 \% suivie de la nécrose de la tête fémorale soit 44,44\%. Elle est surtout due aux salmonelles, contre lesquelles le drépanocytaire se défend mal (Wierenga et coll, 2001). Elle survient toujours au décours d'une crise vaso-occlusive et se développe au sein d'un infarctus osseux. Elle est souvent multiple, au décours de crises multifocales avec plusieurs infarctus (Mabiala, 2006). Ces résultats étaient proches de ceux de SOUNA BS et coll (Souna, 2006) qui avait rapporté une fréquence d'ostéonécrose aigue de 41,9\% ; l'ostéomyélite aiguë $29 \%$ et l'Arthrose 16,1\%. Nos résultats sont différents de ceux rapportés par HOMAWOO K et coll (Homawoo, 1999) qui a Signalé 5 cas d'ostéonécrose aigue ( 3 du fémur et 2 de l'humérus) dans une série de 39 drépanocytaires. La prédominance de l'atteinte de l'os fémoral serait due à la richesse de cet organe en microcirculation.

Les petits vaisseaux sont rapidement obstrués en cas d'hémolyse ayant pour conséquence l'ischémie, l'hypoxie et la nécrose. Cette complication est beaucoup fréquente chez les patients mal ou non suivis qui sont très nombreux en Guinée. La forme drépanocytaire la fréquente était SSFA2 qui a été diagnostiquée chez 14 patients soit 77,77\% des cas. Ce résultat concorde avec les données de la littérature. Dans ces différentes études, la forme SSFA2 était la plus dominante. Cette forme entraine l'hémolyse à tout moment qui aurait provoqué au niveau des petits vaisseaux l'ischémie et la nécrose tissulaire. L'anomalie quantitative de l'hémogramme était dominée par l'anémie rencontrée chez 18 patients soit $100 \%$ des cas suivie d'hyperleucocytose chez 10 patients soit $55,55 \%$ des cas. 


\section{Conclusion}

Il est ressorti de l'étude que la prévalence des complications osseuses est non négligeable. Une étude prospective et analytique avec un échantillon plus élevé serait nécessaire pour identifier les facteurs de risque et élaborer un protocole de prise en charge. Toutes fois, les campagnes de sensibilisation sur la drépanocytose et ses complications seraient indispensables pour leurs préventions.

\section{Remerciements:}

- A tout le personnel du service d'hématologie de l'hôpital national Donka,

- Au personnel du centre national de transfusion sanguine (CNTS) de Guinée

- Au décanat de la faculté des sciences et techniques de la santé (FSTS) de l'Université Gamal Abdel Nasser de Conakry (UGANC)

\section{References:}

1. Cécile J.C : Les enfants avec une drépanocytose - un mémento pour le pédiatre. Paeditrica ; 2012; 23 (5) : 16-19

2. Coulibaly Y, Keita M, Maiga AKM, Guindo Y, Alwata I, Toure AA : Ostéomyélite drépanocytaire au CHU Gabriel Touré ; MALI MED 2010; Tome XXV (4): 29-31

3. Anoosha H: Complications aiguës de la drépanocytose. LA REVUE DU PRATICIEN 2004 ; 54 :1548-1556

4. Catonné Y, Mukisi M, Rouvillain JL, Ribeyre D : Manifestations ostéoarticulaires de la drépanocytose. Maîtrise orthopédique, 2004; 135:1-31

5. Habibi A : Les complications aigues de la drépanocytose. Revue du praticien, 2004; $154: 1548-1556$.

6. N'dri K, Ahoure AM, ZunonKipre E, Konan A, N'zi PK., Etti E et Coll: Aspects radiographiques et tomodensitométriques de la nécrose de la tête fémorale chez le drépanocytaire. Santé-Montrouage 2000 ; $10(1): 27-30$.

7. Wierenga KJJ, Hambleton IR, Wilson RM, Serjeant BE, Serjeant GR : Significance of fever in Jamaican patients with homozygous sickle cell disease. Arch Dis Child, 2001, 84, 156159.

8. Mabiala J : Crise vaso-occlusive drépanocytaire chez l'enfant et l'adolescent à Brazzaville, étude rétrospective de 587 cas. Bull soc path exo, 2005; 98: 365-370.

9. Souna BS, Barchih A, Karadji SA: Les ostéomyélites chez les drépanocytaire: 
10. Aspects épidémiologiques, diagnostiques et thérapeutiques, à propos de 66 cas colliges à NIAMEY ; MALI médicale 2006, XXI(4) ; 21-25

11. Homawoo K. Bissang K, Songne B, Ayite A: Drépanocytose et ostéonécrose de la tête fémorale considérations thérapeutiques à propos de 38 Cas. Med. Afr Noire, 1999 ; 38 ; ; 510-16 